\title{
APPELL POLYNOMIALS AND DIFFERENTIAL EQUATIONS OF INFINITE ORDER
}

\author{
BY \\ J. D. BUCKHOLTZ
}

\begin{abstract}
Let $\Phi(z)=\Sigma_{0}^{\infty} \beta_{j} z^{j}$ have radius of convergence $r(0<r<\infty)$ and no singularities other than poles on the circle $|z|=r$. A complete solution is obtained for the infinite order differential equation (*) $\sum_{0}^{\infty} \beta_{j} u^{(j)}(z)=g(z)$. It is shown that (*) possesses a solution if and only if the function $g$ has a polynomial expansion in terms of the Appell polynomials generated by $\Phi$. The solutions of (*) are expressed in terms of the coefficients which appear in the Appell polynomial expansions of $\mathrm{g}$. An alternate method of solution is obtained, in which the problem of solving (*) is reduced to the problem of finding a solution, within a certain space of entire functions, of a finite order linear differential equation with constant coefficients. Additionally, differential operator techniques are used to study Appell polynomial expansions.
\end{abstract}

1. Introduction. Let $\Phi(z)=\sum_{0}^{\infty} \beta_{j} z^{j}$ have radius of convergence $r(0<r$ $<\infty)$ and no singularities other than poles on the circle $|z|=r$. The differential equation we study is

$$
\Phi(D) u=g
$$

where $D$ denotes the derivative operator and $\Phi(D)$ is the operator which transforms the analytic function $u$ into

$$
\sum_{j=0}^{\infty} \beta_{j} u^{(j)}(z)
$$

We take for the domain of $\Phi(D)$ the family of entire functions $u$ such that (1.2) is uniformly convergent on every compact set. That very little is lost by this requirement is apparent from the following lemma.

Lemma 1.1. If $v$ is analytic on a region $\Omega$ and $\sum_{0}^{\infty} \beta_{j} v^{(j)}(z)$ is uniformly convergent on some open subset of $\Omega$, then $v$ is the restriction to $\Omega$ of an entire function $u$, and $\sum_{0}^{\infty} \beta_{j} u^{(j)}(z)$ is uniformly convergent on every compact set.

In the present paper, we are able to determine both the domain and range of $\Phi(D)$ and to obtain a complete solution of (1.1). There is a deep connection between this problem and the problem of expanding an entire function in an

Received by the editors November 21, 1972.

AMS (MOS) subject classifications (1970). Primary 30A62, 34A35; Secondary 30A16.

Key words and phrases. Appell polynomials, polynomial expansions, infinite order differential equations.

Copyright $\odot 1974$, American Mathematical Society 
infinite series of Appell polynomials. The Appell polynomials generated by the function $\Phi$ are given by

$$
\pi_{k}(z)=\sum_{j=0}^{k} \beta_{k-j} \frac{z^{j}}{j !}, \quad k=0,1,2, \ldots
$$

An entire function $g$ is said to have a $\left\{\pi_{k}\right\}$ expansion if there is a complex sequence $h=\left\{h_{k}\right\}_{0}^{\infty}$ such that $\sum_{k=0}^{\infty} h_{k} \pi_{k}(z)$ is uniformly convergent on compact sets to $g(z)$. We denote by $\varepsilon$ the family of entire functions $g$ which possess $\left\{\pi_{k}\right\}$ expansions.

Let $1 / \alpha_{q}, 1 \leq q \leq \lambda$, denote the (distinct) poles of $\Phi$ on $|z|=r$, and let $m(q)$ denote the order of the pole $1 / \alpha_{q}$. We denote by $H$ the collection of complex sequences $h$ such that each of $\sum_{k=0}^{\infty}\left(\begin{array}{c}k+m(q)-1 \\ m(q)-1\end{array}\right) \alpha_{q}^{k} h_{k}$ converges, $1 \leq q \leq \lambda$, and we let $u$ denote the family of entire functions $u$ such that $\left\{u^{(j)}(0)\right\}_{0}^{\infty}$ belongs to $H$. Note that every member of $u$ has exponential type $r$ or less, and that every function of exponential type less than $r$ belongs to $u$.

Theorem 1.1. The operator $\Phi(D)$ has domain $\mathcal{U}$ and range $\mathcal{\varepsilon}$. If the function $g \in \varepsilon$ has the Appell expansion $g(z)=\sum_{k=0}^{\infty} h_{k} \pi_{k}(z)$, then the function

$$
u(z)=\sum_{k=0}^{\infty} h_{k} \frac{z^{k}}{k !}
$$

satisfies $\Phi(D) u=g$. Conversely, if $g \in \mathcal{E}$ and $u$ satisfies $\Phi(D) u=g$, then $g$ has the Appell expansion

$$
g(z)=\sum_{k=0}^{\infty} u^{(k)}(0) \pi_{k}(z)
$$

Theorem 1.1 reduces the problem of solving $\Phi(D) u=g$ to the problem of expanding $g$ in an Appell series. This problem was solved in [2] and [3]. Before discussing its solution, we consider the homogeneous equation $\Phi(D) u=0$. Let $\left\{w_{i}\right\}_{i=1}^{s}$ denote the zeros (according to multiplicity) of $\Phi$ in the open disk $|z|<r$, and set $T_{0}(z)=\prod_{i=1}^{s}\left(z-w_{i}\right)$, with the convention that $T_{0}(z) \equiv 1$ if $\Phi$ is zero free in $|z|<r$.

Theorem 1.2. $\Phi(D) u=0$ if and only if $T_{0}(D) u=0$.

In view of Theorem 1.1, $u$ satisfies $\Phi(D) u=0$ if and only if $\sum_{k=0}^{\infty} u^{(k)}(0) \pi_{k}(z)$ is uniformly convergent to 0 on every compact set. The Appell series with this property were characterized in [3]. Theorem 1.2 is, therefore, an immediate consequence of Theorem $\mathbf{C}$ of [3].

Let $\Phi(z)=T(z) \varphi(z)$, where $T$ is a polynomial. with no zero outside the closed disk $|z| \leq r$, and $\varphi$ has no zero in $|z| \leq r$. Set

$$
Q(z)=\prod_{q=1}^{\lambda}\left(1-\alpha_{q} z\right)^{\min (m(q), m-1)},
$$


where $m=\max _{1 \leq q \leq \lambda} m(q)$ denotes the largest order of a pole of $\Phi$ on $|z|=r$. We denote by $\exists$ the linear space of entire functions $f$ which satisfy

$$
\lim _{n \rightarrow \infty} r^{-n}\left(D^{n} f\right)(0)=\lim _{n \rightarrow \infty} r^{-n} n^{m-1}\left(D^{n} Q(D) f\right)(0)=0 .
$$

In the special case that $\Phi$ has only simple poles on $|z|=r$, we have $m=1$, $Q(z) \equiv 1$, and $\Im$ is the space of entire functions $f$ which satisfy

$$
f^{(n)}(0)=o\left(r^{n}\right), \quad n \rightarrow \infty .
$$

In general, (1.3) is a necessary condition that $f \in \mathcal{Y}$ and the condition

$$
f^{(n)}(0)=o\left(r^{n} / n^{m-1}\right), \quad n \rightarrow \infty,
$$

is sufficient.

It was shown in [3] that $\varepsilon$ is the image of $\exists$ under the differential operator $T(D)$. In particular, if $\Phi$ is zero free in the closed disk $|z| \leq r$, then $\varepsilon$ is the space 7. We shall prove here that $\varepsilon=\Im$ under the much weaker condition that $\Phi$ has no zero on the circle $|z|=r$. This is an immediate consequence of the following result, together with the observation that $T_{0}=T$ if $\Phi$ has no zero on the circle $|z|=r$.

Theorem 1.3. The operator $T_{0}(D)$ maps $\Im$ onto $\Im$.

Corollary 1.1. Let $T_{1}(z)=T(z) / T_{0}(z)$. The operator $T_{1}(D)$ is a 1-1 linear map of $\exists$ onto $\mathcal{E}$.

As a consequence of Corollary 1.1 , we note that the space $\varepsilon$ is completely determined by the zeros and poles of $\Phi$ on its circle of convergence.

It is not hard to show that $\varepsilon$ is contained in $\mathcal{7}$, and, therefore, that every member of $\varepsilon$ has exponential type $r$ or less. It has been known for a long time [4] that every function of exponential type less than $r$ possesses a $\left\{\pi_{k}\right\}$ expansion. Our differential operator approach yields a slightly stronger version of this important result. Let $d$ denote the largest integer which is the multiplicity of a zero of $T_{1}$ (if $T_{1}$ is constant, we take $d=0$ ).

Theorem 1.4. If $d>0$ and $m=1$, then $\varepsilon$ contains every entire function $g$ such that

$$
\sum_{n=0}^{\infty}\left|g^{(n)}(0)\right| \frac{n^{d-1}}{r^{n}}<\infty
$$

If $m>1$ or $d=0$, then $\varepsilon$ contains every entire function $g$ such that

$$
g^{(n)}(0)=o\left(r^{n} / n^{m+d-1}\right), \quad n \rightarrow \infty .
$$

In either case, $\varepsilon$ contains every entire function $g$ such that

$$
g^{(n)}(0)=o\left(r^{n} / n^{m+d}\right), \quad n \rightarrow \infty .
$$


The results in [2] pertaining to mapping properties of infinite matrices (regarded as sequence-to-sequence operators) possess differential operator analogues. Let $\theta(z)=1 / \varphi(z)=\sum_{j=0}^{\infty} a_{j} z^{j}$, and note that $\theta$ is analytic in the closed disk $|z| \leq r$. This insures that the domain of the differential operator $\theta(D)$ includes all entire functions of exponential type $r$ or less.

Theorem 1.5. The operator $\varphi(D)$ has domain $U$ and is a 1-1 linear map of $U$ onto 7. Its inverse is the restriction of $\theta(D)$ to 7 . The operator $\Phi(D)$ has domain $U$ and satisfies $\Phi(D) u=T(D)\{\varphi(D) u\}$ for every $u \in \mathcal{U}$.

As a consequence of Theorem 1.5, we see that the differential equation $\varphi(D) u=f$ has a solution if and only if $f \in \mathcal{Y}$. If $f \in \mathcal{Y}$, then one obtains the unique solution of

$$
\varphi(D) u=f
$$

by "dividing" both sides of (1.4) by $\varphi(D)$, i.e., $u=\theta(D) f$.

Theorem 1.5 also allows us to obtain a solution of $\Phi(D) u=g$ by reducing it to a finite order linear differential equation with constant coefficients. The following is an easy consequence of Theorem 1.5.

Theorem 1.6. Suppose that $g$ is an entire function. The infinite order differential equation

$$
\Phi(D) u=g
$$

has a solution if and only if the differential equation

$$
T(D) f=g
$$

has a solution $f$ which belongs to $\Im$. If $f \in \mathcal{Y}$ and $T(D) f=g$, then $u=\theta(D) f$ is $a$ solution of (1.5). Conversely, if $u$ is a solution of (1.5), then the function $f=\varphi(D) u$ belongs to $\exists$, satisfies $T(D) f=g$, and has the property that $u=\theta(D) f$.

Theorem 1.6, while very nearly a restatement of Theorem 1.1, does not depend on Appell polynomials, and is, to that extent, a more "natural" solution of (1.5).

2. Proofs of Theorems 1.1 and 1.3. It was established in [2] that a sequence $h$ belongs to $H$ if and only if each of the series $\sum_{j=k}^{\infty} \beta_{j-k} h_{j}, 0 \leq k<\infty$, converges (the additional hypothesis in [2] that $\Phi$ have no zeros in the disk $|z| \leq r$ was not used in the proof of this).

Lemma 2.1. Suppose $v$ is analytic on some region containing the complex number $w$, and

$$
\sum_{j=0}^{\infty} \beta_{j} v^{(j)}(z)
$$

is uniformly convergent on a neighborhood of $w$. Then the sequence $\left\{v^{(j)}(w)\right\}_{0}^{\infty}$ belongs to $H$, and $v$ is the restriction of an entire function. 
Proof. Let $k$ denote a nonnegative integer, differentiate (2.1) $k$ times, and set $z=w$. Therefore

$$
\sum_{j=0}^{\infty} \beta_{j} v^{(j+k)}(w)=\sum_{j=k}^{\infty} \beta_{j-k} v^{(j)}(w)
$$

converges, so that $\left\{v^{(j)}(w)\right\}_{0}^{\infty}$ belongs to $H$. From the definition of $H$, we obtain

$$
\limsup _{j \rightarrow \infty}\left|v^{(j)}(w)\right|^{1 / j} \leq r .
$$

Therefore the series

$$
u(z)=\sum_{j=0}^{\infty} v^{(j)}(w) \frac{(z-w)^{j}}{j !}
$$

defines an entire function $u$ which agrees with $v$ on a neighborhood of $w$. Note that (2.2) implies that $u$ is of exponential type $r$ or less.

Theorem 2.1. If $u$ is entire, $w$ is a complex number, and $\left\{u^{(j)}(w)\right\}_{0}^{\infty}$ belongs to $H$, then

$$
\sum_{j=0}^{\infty} \beta_{j} u^{(j)}(z)=\sum_{k=0}^{\infty} u^{(k)}(w) \pi_{k}(z-w)
$$

for all $z$, and both series are uniformly convergent on every compact set.

Proof. It is no loss of generality to take $w=0$. To see this, replace $z$ by $z+w$ and let $u_{1}(z)=u(z+w)$.

Taking $w=0$, we have that $\left\{u^{(j)}(w)\right\}_{0}^{\infty}=\left\{u^{(j)}(0)\right\}_{0}^{\infty}$ belongs to $H$, and it follows from this [2] that $\sum_{k=0}^{\infty} u^{(k)}(0) \pi_{k}(z)$ is uniformly convergent on compact sets to an entire function $g$. To complete the proof, we show that

$$
\lim _{n \rightarrow \infty}\left\{g(z)-\sum_{j=0}^{n} \beta_{j} u^{(j)}(z)\right\}=0
$$

uniformly on compact sets.

For notational simplicity, set $h_{k}=u^{(k)}(0)$. We have

$$
\begin{aligned}
\sum_{j=0}^{n} \beta_{j} u^{(j)}(z) & =\sum_{j=0}^{n} \beta_{j} \sum_{k=j}^{\infty} h_{k} \frac{z^{k-j}}{(k-j) !} \\
& =\sum_{k=0}^{\infty} h_{k} \sum_{j=0}^{\min \{n, k\}} \beta_{j} \frac{z^{k-j}}{(k-j) !} \\
& =\sum_{k=0}^{n} h_{k} \sum_{j=0}^{k} \beta_{j} \frac{z^{k-j}}{(k-j) !}+\sum_{k=n+1}^{\infty} h_{k} \sum_{j=0}^{n} \beta_{j} \frac{z^{k-j}}{(k-j) !} \\
& =\sum_{k=0}^{n} h_{k} \pi_{k}(z)+\sum_{k=n+1}^{\infty} h_{k} \sum_{j=0}^{n} \beta_{j} \frac{z^{k-j}}{(k-j) !} .
\end{aligned}
$$


Therefore

$$
\begin{aligned}
g(z)-\sum_{j=0}^{n} \beta_{j} u^{(j)}(z) & =\sum_{k=n+1}^{\infty} h_{k}\left\{\pi_{k}(z)-\sum_{j=0}^{n} \beta_{j} \frac{z^{k-j}}{(k-j) !}\right\} \\
& =\sum_{k=n+1}^{\infty} h_{k} \sum_{j=n+1}^{k} \beta_{j} \frac{z^{k-j}}{(k-j) !} .
\end{aligned}
$$

To estimate this quantity, we use the poles of $\Phi$ on $|z|=r$ to obtain an asymptotic estimate for $\beta_{j}$. For $1 \leq q \leq \lambda$, let

$$
\sum_{i=1}^{m(q)} \frac{c_{t}(q)}{\left(1-\alpha_{q} z\right)^{m(q)+1-t}}
$$

denote the sum of the negative powers in the Laurent expansion of $\Phi$ at $\alpha_{q}^{-1}$. Then

$$
\Phi(z)=\sum_{q=1}^{\lambda} \sum_{t=1}^{m(q)} \frac{c_{t}(q)}{\left(1-\alpha_{q} z\right)^{m(q)+1-t}}+\Phi_{2}(z),
$$

where $\Phi_{2}(z)=\sum_{j=0}^{\infty} \beta_{j}^{\prime} z^{j}$ is analytic in a disk $|z| \leq r_{1}, r_{1}>r$. We then have

$$
\beta_{j}=\sum_{q=1}^{\lambda} \sum_{t=1}^{m(q)} c_{t}(q)\left(\begin{array}{c}
j+m(q)-t \\
m(q)-t
\end{array}\right) \alpha_{q}^{j}+\beta_{j}^{\prime}
$$

Therefore

$$
\sum_{k=n+1}^{\infty} h_{k} \sum_{j=n+1}^{k} \beta_{j} \frac{z^{k-j}}{(k-j) !}=R_{n}(z)+\sum_{q=1}^{\lambda} \sum_{j=1}^{m(q)} U_{q t n}(z),
$$

where $R_{n}(z)=\sum_{k=n+1}^{\infty} h_{k} \sum_{j=n+1}^{k} \beta_{j}^{\prime} z^{k-j} /(k-j)$ ! and

$$
U_{q t n}(z)=\sum_{k=n+1}^{\infty} h_{k} \sum_{j=n+1}^{k}\left(\begin{array}{c}
j+m(q)-t \\
m(q)-t
\end{array}\right) \alpha_{q}^{j} \frac{z^{k-j}}{(k-j) !} .
$$

The proof that $\lim _{n \rightarrow \infty} R_{n}(z)=0$ uniformly on compact sets is straightforward and is omitted. The following lemma completes the proof of Theorem 2.1.

Lemma 2.2. If $1 \leq q \leq \lambda$ and $1 \leq t \leq m(q)$, then $\lim _{n \rightarrow \infty} U_{q t n}(z)=0$ uniformly on compact sets.

Proof of Lemma 2.2. From [2, Lemma 2.7] we have

$$
\left|U_{q I n}(z)\right| \leq V(z) \sup _{0 \leq i<\infty}\left|\sum_{k=n+1+i}^{\infty}\left(\begin{array}{c}
k+m(q)-1 \\
m(q)-1
\end{array}\right) \alpha_{q}^{k} h_{k}\right|,
$$

where $V(z)$ denotes the total variation of the sequence $\left\{y_{k}\right\}_{k=n+1}^{\infty}$ given by

$$
y_{k}=\sum_{j=n+1}^{k}\left(\begin{array}{c}
j+m(q)-t \\
m(q)-t
\end{array}\right)\left(\begin{array}{c}
k+m(q)-1 \\
m(q)-1
\end{array}\right)^{-1} \frac{\left(z / \alpha_{q}\right)^{k-j}}{(k-j) !}
$$


Replace the summation index $j$ by $k-j$ and set $y_{k}^{\prime}=y_{k+n+1}$. After some simplification, one obtains

$$
y_{k}^{\prime}=\sum_{j=0}^{k}\left(\begin{array}{c}
k+n+1-j+m(q)-t \\
m(q)-t
\end{array}\right)\left(\begin{array}{c}
k+n+m(q) \\
m(q)-1
\end{array}\right)^{-1} \frac{\left(z / \alpha_{q}\right)^{j}}{j !} .
$$

The sequence $\left\{y_{k}^{\prime}\right\}_{k=0}^{\infty}$ has total variation $V(z)$; also, $\left|y_{0}^{\prime}\right|=\left(\begin{array}{c}n+1+m(q)-1 \\ m(q)-1\end{array}\right)\left(\begin{array}{c}n+m(q) \\ m(q)-1\end{array}\right)^{-1}$ $\leq 1$. Set

$$
x_{k j}=\left(\begin{array}{c}
k+n+1-j+m(q)-t \\
m(q)-t
\end{array}\right)\left(\begin{array}{c}
k+n+m(q) \\
m(q)-1
\end{array}\right)^{-1}, \quad 0 \leq j \leq k<\infty .
$$

Then

$$
y_{k}^{\prime}-y_{k+1}^{\prime}=\sum_{j=0}^{k}\left(x_{k j}-x_{k+1, j}\right) \frac{\left(z / \alpha_{q}\right)^{j}}{j !}-x_{k+1, k+1} \frac{\left(z / \alpha_{q}\right)^{k+1}}{(k+1) !}
$$

and

$$
\left|x_{k+1, k+1} \frac{\left(z / \alpha_{q}\right)^{k+1}}{(k+1) !}\right| \leq \frac{\left|z / \alpha_{q}\right|^{k+1}}{(k+1) !}
$$

Therefore

$$
\begin{aligned}
V(z) & =\left|y_{0}^{\prime}\right|+\sum_{k=0}^{\infty}\left|y_{k}^{\prime}-y_{k+1}^{\prime}\right| \\
& \leq 1+\sum_{k=0}^{\infty} \frac{\left|z / \alpha_{q}\right|^{k+1}}{(k+1) !}+\sum_{k=0}^{\infty} \sum_{j=0}^{k}\left|x_{k j}-x_{k+1, j}\right| \frac{\left|z / \alpha_{q}\right|^{\prime}}{j !} \\
& =\exp \left|z / \alpha_{q}\right|+\sum_{j=0}^{\infty} \frac{\left|z / \alpha_{q}\right|^{j}}{j !} \sum_{k=j}^{\infty}\left|x_{k j}-x_{k+1, j}\right|
\end{aligned}
$$

The method used to prove Lemma 3.2 of [2] allows one to establish that $\sum_{k=j}^{\infty}\left|x_{k j}-x_{k+1, j}\right| \leq 2$. Therefore $V(z) \leq 3 \exp \left|z / \alpha_{q}\right|=3 e^{|r z|}$. This completes the proof of Lemma 2.2 and also the proof of Theorem 2.1. Note that Lemma 1.1 is a consequence of Lemma 2.1 and Theorem 2.1.

Proof of Theorem 1.1. It follows from Theorem 2.1 (with $w=0$ ) that $u$ is contained in the domain of $\Phi(D)$, and from Lemma 2.1 (with $w=0$ ) that $u$ contains the domain of $\Phi(D)$. Therefore the domain of $\Phi(D)$ is $u$; using Theorem 2.1 again, we see that the range of $\Phi(D)$ is contained in $\varepsilon$. Suppose now that $g \in \varepsilon$. Then there is a complex sequence $h$ such that $g(z)=\sum_{k=0}^{\infty} h_{k} \pi_{k}(z)$ uniformly on compact sets. From the Convergence Theorem in [2] it follows that $h \in H$. Consequently the function $u(z)=\sum_{k=0}^{\infty} h_{k} z^{k} / k$ ! belongs to $u$, and from Theorem 2.1 we have

$$
(\Phi(D) u)(z)=\sum_{k=0}^{\infty} u^{(k)}(0) \pi_{k}(z)=\sum_{k=0}^{\infty} h_{k} \pi_{k}(z)=g(z) .
$$


Therefore the range of $\Phi(D)$ is $\varepsilon$. Suppose now that $g \in \mathcal{E}$ and that $u$ satisfies $\Phi(D) u=g$. From Theorem 2.1 we obtain

$$
g(z)=(\Phi(D) u)(z)=\sum_{k=0}^{\infty} u^{(k)}(0) \pi_{k}(z)
$$

and the proof of Theorem 1.1 is complete.

Proof of Theorem 1.3. If $T_{0}(z) \equiv 1$, there is nothing to prove, since in this case $T_{0}(D)=I$, the identity operator. If $T_{0}(z)=\prod_{i=1}^{s}\left(z-w_{i}\right), s \geq 1$, we have the operator factorization $T_{0}(D)=\prod_{i=1}^{s}\left(D-w_{i} I\right)$, so that it is sufficient to prove that, if $\left|w_{0}\right|<r$, then $D-w_{0} I$ maps $\Im$ onto $\Im$. Clearly $D-w_{0} I$ maps $\Im$ into $\Im$, since $\mathcal{Y}$ is a linear space which is closed under differentiation. If $f \in \mathcal{Y}$, then so does $\sum_{j=1}^{\infty} f^{(j-1)}(0) z^{j} / j$ !; therefore we can exclude the case $w_{0}=0$ and reduce the problem to that of showing that every $F \in \mathcal{Y}$ is the image of some $f \in \mathcal{Y}$ under the transformation $w D-I$, where $w$ is a complex constant such that $|w|>1 / r$.

Suppose that $F \in \dot{\mathcal{Y}}$; the differential equation with boundary condition

$$
w f^{\prime}-f=F, \quad f(0)=0,
$$

has a unique solution $f$. We shall show that this function belongs to 7 . Set $Q(z)=\sum_{t=0}^{r} C_{t} z^{t}$; it is easy to verify that a function $g$ belongs to $\exists$ if and only if

$$
\lim _{n \rightarrow \infty} r^{-n} g^{(n)}(0)=\lim _{n \rightarrow \infty} n^{m-1} r^{-n} \sum_{t=0}^{+} C_{t} g^{(n+t)}(0)=0 .
$$

Let $k$ denote a positive integer, differentiate (2.3) $k$ times, multiply by $w^{k}$, and set $z=0$. This yields

$$
w^{k+1} f^{(k+1)}(0)-w^{k} f^{(k)}(0)=w^{k} F^{(k)}(0)
$$

Since $f(0)=0$, we have $w f^{\prime}(0)=F(0)$, which corresponds to $k=0$ in (2.5). If we sum equation (2.5) over $0 \leq k<n$, we obtain

$$
w^{n} f^{(n)}(0)=\sum_{k=0}^{n-1} w^{k} F^{(k)}(0)
$$

Therefore the solution of (2.3) satisfies

$$
f^{(n)}(0)=\sum_{k=0}^{n-1} w^{k-n} F^{(k)}(0), \quad 1 \leq n<\infty .
$$

To complete the proof, we use the fact that (2.4) holds if $g=F$ to prove that (2.4) holds if $g=f$.

We have

$$
r^{-n} f^{(n)}(0)=\sum_{k=0}^{n-1}(r w)^{k-n} r^{-k} F^{(k)}(0)
$$


Since $|r w|>1$, the infinite matrix

$$
\begin{aligned}
M_{n k} & =(r w)^{k-n}, & & 0 \leq k<n, \\
& =0, & & k \geq n,
\end{aligned}
$$

transforms sequences with limit 0 into sequences with limit 0 . Therefore $\lim _{k \rightarrow \infty} r^{-k} F^{(k)}(0)=0$ implies $\lim _{n \rightarrow \infty} r^{-n} f^{(n)}(0)=0$.

The remainder of the proof is similar in nature but more difficult. We again use the fact that sufficient (and also necessary) conditions for an infinite matrix $N$ to transform sequences with limit 0 into sequences with limit 0 are that

$$
\lim _{n \rightarrow \infty} N_{n k}=0, \quad k=0,1,2, \ldots
$$

and

$$
\sup _{0 \leq n<\infty} \sum_{k=0}^{\infty}\left|N_{n k}\right|<\infty
$$

We have

$$
n^{m-1} r^{-n} \sum_{t=0}^{+} C_{t} f^{(n+t)}(0)=n^{m-1} r^{-n} \sum_{t=0}^{+} C_{t} \sum_{k=0}^{n+t-1} w^{k-n-t} F^{(k)}(0)
$$

Now

$$
\begin{aligned}
\sum_{t=0}^{\tau} C_{t} \sum_{k=0}^{n+t-1} w^{k-n-t} F^{(k)}(0)=\sum_{t=0}^{\tau} C_{t} \sum_{k=-t}^{n-1} w^{k-n} F^{(k+t)}(0) \\
=\sum_{t=0}^{\tau} C_{t} \sum_{k=-t}^{0} w^{k-n} F^{(k+t)}(0)+\sum_{t=0}^{\tau} C_{t} \sum_{k=1}^{n-1} w^{k-n} F^{(k+t)}(0) .
\end{aligned}
$$

It is easy to show that

$$
\lim _{n \rightarrow \infty} n^{m-1} r^{-n} \sum_{t=0}^{\tau} C_{t} \sum_{k=-t}^{0} w^{k-n} F^{(k+t)}(0)=0
$$

Now

$$
\begin{aligned}
n^{m-1} r^{-n} \sum_{t=0}^{\tau} C_{t} \sum_{k=1}^{n-1} & w^{k-n} F^{(k+t)}(0)=n^{m-1}(r w)^{-n} \sum_{k=1}^{n-1} w^{k} \sum_{t=0}^{+} C_{t} F^{(k+\imath)}(0) \\
= & \frac{n^{m-1}}{(r w)^{n}} \sum_{k=1}^{n-1} \frac{(r w)^{k}}{k^{m-1}} k^{m-1} r^{-k} \sum_{t=0}^{\tau} C_{t} F^{(k+t)}(0) \\
= & \sum_{k=1}^{n-1}(r w)^{k-n}\left(\frac{n}{k}\right)^{m-1}\left\{k^{m-1} r^{-k} \sum_{t=0}^{T} C_{t} F^{(k+t)}(0)\right\} .
\end{aligned}
$$

To complete the proof, it suffices to show that the infinite matrix 


$$
\begin{aligned}
N_{n k} & =0 & & \text { if } k=0 \text { or } k \geq n, \\
& =(n w)^{k-n}(n / k)^{m-1} & & \text { if } 1<k<n,
\end{aligned}
$$

has properties (i) and (ii). Property (i) is obvious. To establish (ii), set $\rho=|r w|^{-1}$ $<1$. Then

$$
\begin{aligned}
\sum_{k=0}^{\infty}\left|N_{n k}\right| & =\sum_{k=1}^{n-1} \rho^{n-k}\left(\frac{n}{k}\right)^{m-1} \\
& =\sum_{k=1}^{n-1} \rho^{k}\left(\frac{n}{n-k}\right)^{m-1}=\sum_{k=1}^{n-1} k^{m-1} \rho^{k}\left\{\frac{n}{k(n-k)}\right\}^{m-1} .
\end{aligned}
$$

Since $k(n-k)$ is least at $k=1$ and $k=n-1$, we have

$$
\sum_{k=0}^{\infty}\left|N_{n k}\right| \leq\left(\frac{n}{n-1}\right)^{m-1} \sum_{k=1}^{n-1} k^{m-1} \rho^{k} \leq 2^{m-1} \sum_{k=1}^{\infty} k^{m-1} \rho^{k}
$$

and this completes the proof.

Proof of Corollary 1.1. The operator $T(D)$ maps $\rightarrow$ onto $\varepsilon$ and has the factorization $T(D)=T_{1}(D)\left\{T_{0}(D)\right\}$. In view of Theorem 1.3, we need only show that the restriction of $T_{1}(D)$ to $\mathcal{Y}$ is $1-1$. If $T_{1}(z)$ is a constant, there is nothing to prove. If not, $T_{1}(D)$ has a factorization into differential operators of order 1 . It is easily verified that these differential operators are 1-1 on 9 , and this completes the proof.

3. Proofs of Theorems 1.5 and 1.6. Let $v(z)=v_{1}(z) v_{2}(z)$, where $v_{1}$ and $v_{2}$ are functions analytic in a neighborhood of 0 . For convenience, we shall sometimes use $v_{1}(D) v_{2}(D)$ to denote the operator $v(D)$. Since we shall always denote operator composition by $v_{1}(D)\left\{v_{2}(D)\right\}$, no confusion will result.

Lemma 3.1. If $v_{1}$ and $v_{2}$ are functions analytic in the closed disk $|z| \leq r$, then $v_{1}(D)\left\{v_{2}(D) f\right\}=\left\{v_{1}(D) v_{2}(D)\right\} f$ for every entire function $f$ of exponential type $r$ or less.

Proof. Suppose that $f$ is of exponential type $r$ or less. For $\delta>0$ we have

$$
\limsup _{j \rightarrow \infty}\left|f^{(j)}(0)\right|^{p / j} \leq r<r(1+\delta)
$$

Therefore the quantity $K_{\delta}=\sup _{0 \leq j<\infty}\left|f^{(j)}(0)\right| /\{r(1+\delta)\}^{j}$ is finite. Consequently,

$$
\begin{aligned}
\left|f^{(k)}(z)\right| & \leq \sum_{j=k}^{\infty}\left|f^{(j)}(0)\right| \frac{|z|^{j-k}}{(j-k) !} \\
& \leq K_{\delta}\{r(1+\delta)\}^{k} \sum_{j=k}^{\infty}\{r(1+\delta)\}^{j-k} \frac{|z|^{j-k}}{(j-k) !} \\
& =K_{\delta}\{r(1+\delta)\}^{k} \exp \{r(1+\delta)|z|\} .
\end{aligned}
$$


Suppose that $v$ is analytic in the closed disk $|z| \leq r$. Choose $\delta>0$ so that $v$ is analytic in the closed disk $|z| \leq r(1+\delta)$. The previous estimate insures that

$$
\{v(D) f\}(z)=\sum_{k=0}^{\infty} \frac{v^{(k)}(0)}{k !} f^{(k)}(z)
$$

is uniformly convergent on every compact set. Note also that

$$
|\{v(D) f\}(z)| \leq K_{\delta} \exp \{r(1+\delta)|z|\} \sum_{k=0}^{\infty} \frac{\left|v^{(k)}(0)\right|}{k !}\{r(1+\delta)\}^{k},
$$

so that $v(D) f$ is of exponential type $r(1+\delta)$ or less; consequently $v(D) f$ is of exponential type $r$ or less since $\delta$ is arbitrary.

Now

$$
\begin{aligned}
\left\{v_{1}(D)\left\{v_{2}(D) f\right\}\right\}(z) & =\sum_{j=0}^{\infty} \frac{v_{1}^{(j)}(0)}{j !} \sum_{k=0}^{\infty} \frac{v_{2}^{(k)}(0)}{k !} f^{(k+j)}(z) \\
& =\sum_{j=0}^{\infty} \frac{v_{1}^{(j)}(0)}{j !} \sum_{k=j}^{\infty} \frac{v_{2}^{(k-j)}(0)}{(k-j) !} f^{(k)}(z) \\
& =\sum_{k=0}^{\infty}\left\{\sum_{j=0}^{k} \frac{v_{1}^{(j)}(0)}{j !} \frac{v_{2}^{(k-j)}(0)}{(k-j) !}\right\} f^{(k)}(z) \\
& =\left\{\left\{v_{1}(D) v_{2}(D)\right\} f\right\}(z),
\end{aligned}
$$

provided that the interchange in order of summation is valid. To see that this is the case, note that

$$
\sum_{k=0}^{\infty}\left\{\sum_{j=0}^{k} \frac{\left|v_{1}^{(j)}(0)\right|}{j !} \frac{\left|v_{2}^{(k-j)}(0)\right|}{(k-j) !}\right\}\left|f^{(k)}(z)\right|
$$

does not exceed

$$
K_{\delta} \exp \{r(1+\delta)|z|\} \sum_{k=0}^{\infty}\left\{\sum_{j=0}^{k} \frac{\left|v_{1}^{(j)}(0)\right|}{j !} \frac{\left|v_{2}^{(k-j)}(0)\right|}{(k-j) !}\right\}\{r(1+\delta)\}^{k},
$$

which is finite, provided that $\delta$ is chosen so that both $v_{1}$ and $v_{2}$ are analytic in the closed disk $|z| \leq r(1+\delta)$.

Lemma 3.2. If $v_{1}$ is polynomial and $v_{2}$ is analytic in a neighborhood of 0 , then $v_{1}(D)\left\{v_{2}(D) f\right\}=\left\{v_{1}(D) v_{2}(D)\right\} f$ for every $f$ in the domain of $v_{2}(D)$.

Proof. The interchange in order of summation is trivial to verify in this case, since the "outer" sum is finite.

Theorem 3.1. If $u \in U$, then $\theta(D)\{\varphi(D) u\}=u$.

Proof. Set $P(z)=\prod_{q=1}^{\lambda}\left(1-\alpha_{q} z\right)^{m(q)}$ and note that the functions $v_{1}=\theta / P$, $v_{2}=P$, and $v_{3}=P \varphi$ are analytic in the closed disk $|z| \leq r$. In the proof of 
Theorem 1.1 the possibility that $T(z) \equiv 1$ is not excluded. Therefore $\varphi(D)$ has domain $u$ and range 3 . From Lemma 3.2 we have $P(D)\{\varphi(D) u\}=v_{3}(D) u$. The function $f=\varphi(D) u$ is of exponential type $r$ or less; from Lemma 3.1 we obtain

$$
v_{1}(D)\{P(D) f\}=\theta(D) f=\theta(D)\{\varphi(D) u\} .
$$

Since $u$ is of exponential type $r$ or less, we have

$$
\begin{aligned}
\theta(D)\{\varphi(D) u\} & =v_{1}(D)\{P(D) f\}=v_{1}(D)\{P(D)\{\varphi(D) u\}\} \\
& =v_{1}(D)\left\{v_{3}(D) u\right\}=\left\{v_{1}(D) v_{3}(D)\right\} u=u
\end{aligned}
$$

Proof of Theorem 1.5. Most of Theorem 1.5 follows from Theorem 1.1, since in Theorem 1.1 the case $T(z) \equiv 1$ is not excluded, and in this case $\Phi=\varphi$. The factorization $\Phi(D) u=T(D)\{\varphi(D) u\}$ is a consequence of Lemma 3.1. That $\varphi(D)$ is $1-1$ follows from Theorem 3.1, as does the assertion that the inverse of $\varphi(D)$ is the restriction to $\rightarrow$ of $\theta(D)$.

Proof of Theorem 1.6. The second sentence of Theorem 1.6 is equivalent to the assertion that $\varepsilon$ is the range of $\Phi(D)$, and this has already been established. Suppose that $f \in \mathcal{Y}$ and $T(D) f=g$. Set $u=\theta(D) f$. From Theorem 1.5 we have $u \in U$ and $\Phi(D) u=T(D)\{\varphi(D) u\}=T(D) f=g$.

Suppose now that $u$ is such that $\Phi(D) u=g$. From Theorem 1.1 we have $u \in U$. Therefore the function $f=\varphi(D) u$ belongs to $\rightarrow$ by Theorem 1.5. Also from Theorem 1.5 we obtain $u=\theta(D) f$ and

$$
g=\Phi(D) u=T(D)\{\varphi(D) u\}=T(D) f,
$$

which completes the proof.

4. Proof of Theorem 1.4. Suppose that $m=1$ and $d>0$. Set

$$
S(z)=\frac{1}{T_{1}(z)}=\sum_{j=0}^{\infty} s_{j} z^{j}
$$

Since $S$ is zero free and has only poles on its circle of convergence, the operators $S(D)$ and $T_{1}(D)$ are controlled by Theorem 1.5. Let $u(S)$ and $\Im(S)$ denote the spaces obtained from $U$ and $\Im$ by replacing $\Phi$ by $S$. Since $m=1, \Im$ is the collection of all entire $f$ such that

$$
f^{(n)}(0)=o\left(r^{n}\right), \quad n \rightarrow \infty .
$$

Every member of $\Im(S)$ must satisfy (4.1) and one other growth condition; therefore $\Im(S)$ is contained in $\Im$. If one writes out explicitly the condition that a function $g$ belongs to $\mathcal{U}(S)$, it follows easily that $\mathcal{U}(S)$ contains every entire $g$ such that

$$
\sum_{n=0}^{\infty}\left|g^{(n)}(0)\right| \frac{n^{d-1}}{r^{n}}<\infty .
$$


From Theorem 1.5 we see that $T_{1}(D)$ maps $\vartheta(S)$ onto $U(S)$. Therefore every $g$ which satisfies (4.2) is the image under $T_{1}(D)$ of an $f$ which belongs to $\Im(S)$, and therefore to $\exists$. Since $\varepsilon$ is the image of $\Im$ under $T_{1}(D)$, the proof of the first half is complete.

Suppose that $m>1$. If $d=0$, there is nothing to prove, since in this case $\varepsilon=\exists$. Since no pole of $S$ on $|z|=r$ is of order greater than $d$, we have the estimate

$$
S^{(j)}(0) / j !=s_{j}=O\left(j^{d-1} / r^{j}\right), \quad j \rightarrow \infty .
$$

Since $m \geq 2$, the growth condition $g^{(j)}(0)=o\left(r^{j} / j^{m+d-1}\right), j \rightarrow \infty$, implies

$$
g^{(j)}(0)=o\left(r^{j} / j^{d+1}\right), \quad j \rightarrow \infty .
$$

We see from Theorem 1.1 that (4.3) is sufficient to guarantee that $g$ belongs to $u(S)$, the domain of $S(D)$. Set $f=S(D) g$. From Lemma 3.2 we have $g$ $=T_{1}(D)\{S(D) g\}=T_{1}(D) f$. To complete the proof, we need only show that $f \in \mathcal{Y}$. We establish this by showing that $f^{(n)}(0)=o\left(r^{n} / n^{m-1}\right), n \rightarrow \infty$.

We have

$$
f^{(n)}(0)=(S(D) g)^{(n)}(0)=\sum_{j=n}^{\infty} s_{j-n} g^{(j)}(0)
$$

Therefore

$$
\begin{aligned}
\left|r^{-n} f^{(n)}(0)\right| & =\left|\sum_{j=n}^{\infty}\left(\begin{array}{c}
j+m \\
m
\end{array}\right) g^{(j)}(0) s_{j-n} r^{-n}\left(\begin{array}{c}
j+m \\
m
\end{array}\right)^{-1}\right| \\
& \leq\left\{\sup _{j \geq n}\left|\left(\begin{array}{c}
j+m \\
m
\end{array}\right) g^{(j)}(0) s_{j-n} r^{-n}\right|\right\} \sum_{j=n}^{\infty}\left(\begin{array}{c}
j+m \\
m
\end{array}\right)^{-1} \\
& =\frac{m}{m-1}\left(\begin{array}{c}
n+m-1 \\
m-1
\end{array}\right)^{-1} \sup _{j \geq n}\left|\left(\begin{array}{c}
j+m \\
m
\end{array}\right) g^{(j)}(0) s_{j-n} r^{-n}\right| .
\end{aligned}
$$

There exist constants $K_{1}$ and $K_{2}$ such that, if $1 \leq n \leq j$, then

$$
\left(\begin{array}{c}
j+m \\
m
\end{array}\right) \leq K_{1} j^{m} \text { and }\left|s_{j-n}\right| \leq K_{2} j^{d-1} / r^{j-n} .
$$

Therefore

$$
\left|\left(\begin{array}{c}
++m \\
m
\end{array}\right) g^{(j)}(0) s_{j-n} r^{-n}\right| \leq K_{1} K_{2} \frac{j^{m+d-1}}{r^{j}}\left|g^{(j)}(0)\right|
$$

and we have

$$
\left(\begin{array}{c}
n+m-1 \\
m-1
\end{array}\right) r^{-n}\left|f^{(n)}(0)\right| \leq \frac{K_{1} K_{2} m}{m-1} \sup _{j \geq n}\left|\frac{j^{m+d-1}}{r^{j}} g^{(j)}(0)\right| .
$$


Since $\lim _{j \rightarrow \infty}\left(j^{m+d-1} / r^{j}\right) g^{(j)}(0)=0$, the proof is complete.

5. An example. Take $\Phi(z)=(1+z)^{2} /(1-z)$. Then $\Im$ is the set of entire $f$ such that $f^{(n)}(0)=o(1), n \rightarrow \infty$, and

$$
\pi_{k}(z)=4 \sum_{j=0}^{k-2} z^{j} / j !+\frac{3 z^{k-1}}{(k-1) !}+\frac{z^{k}}{k !} .
$$

It follows from the Convergence Theorem in [2] that convergence of $\sum_{k=0}^{\infty} h_{k} \pi_{k}(z)$ for one value of $z$ implies uniform convergence on every compact set. The sequence $\left\{\pi_{k}\right\}_{0}^{\infty}$ is biorthogonal to the sequence of linear functionals $\left\{\mathcal{L}_{k}\right\}_{0}^{\infty}$ given by

$$
\mathcal{L}_{k}(g)=\sum_{j=k}^{\infty}(-1)^{j-k}(2 j-2 k+1) g^{(j)}(0), \quad k=0,1,2, \cdots
$$

Every entire $g$ for which the functionals $\mathcal{L}_{k}(g)$ are defined has the formal basic series expansion

$$
g(z) \sim \sum_{k=0}^{\infty} \mathcal{L}_{k}(g) \pi_{k}(z)
$$

(We use the term "basic series" in the sense of Whittaker [5].) It follows from [3, Theorem C] that the $\left\{\pi_{k}\right\}$ expansions are unique. In spite of this, not every convergent $\left\{\pi_{k}\right\}$ expansion is a basic series expansion. To see this, take

$$
g(z)=\sum_{j=0}^{\infty}\left(2 j^{2}+8 j+7\right) \frac{z^{j}}{(j+3) !}
$$

and note that $g=f^{\prime \prime}+2 f^{\prime}+f$, where $f(z)=\left(e^{z}-1\right) / 2 z$. Since $f \in \mathcal{Y}$, it follows from [3, Theorem B] that $g$ has the convergent $\left\{\pi_{k}\right\}$ expansion

$$
g(z)=\sum_{k=0}^{\infty}\left\{f^{(k)}(0)-f^{(k+1)}(0)\right\} \pi_{k}(z)=\sum_{k=0}^{\infty} \frac{\pi_{k}(z)}{2(k+1)(k+2)} .
$$

On the other hand, the series defining $\mathcal{L}_{k}(g)$ is easily seen to be divergent for every $k, 0 \leq k<\infty$.

\section{REFERENCES}

1. R. P. Boas and R. C. Buck, Polynomial expansions of analytic functions, 2nd ed., Ergebnisse der Mathematik und ihrer Grenzgebiete, N.F., Band 19, Academic Press, New York; Springer-Verlag, Berlin, 1964. MR 29 \#218.

2. J. D. Buckholtz, Appell polynomial expansions and biorthogonal expansions in Banach spaces, Trans. Amer. Math. Soc. (to appear).

3.—. Appell polynomials whose generating function is meromorphic on its circle of convergence, Bull. Amer. Math. Soc. 79 (1973), 469-472.

4. W. T. Martin, On expansions in terms of a certain general class of functions, Amer. J. Math. 58 (1936), 407-420.

5. J. M. Whittaker, Interpolatory function theory, Cambridge Univ. Press, Cambridge, 1935.

Department of Mathematics, University of Kentucky, LeXington, Kentucky 40506 